\title{
Análisis Computacional de Modelos Biológicos para su Aplicación a Modelos Económicos
}

\author{
Roberto R. Gómez, Guillermo S. Navas, Patricia Cuadros y Graciela B. Ganyitano \\ Universidad Nacional de San Juan, Facultad de Ingeniería, Departamento de Matemáticas, \\ Av. Libertador San Martín 1109(O), Capital, (5400) San Juan-Argentina \\ (e-mail: snavas@unsj.edu.ar)
}

\section{Resumen}

Se examina la vinculación que existe entre la dinámica de la economía y la de las poblaciones ecológicas y se realiza una aplicación educativa. Se propone un modelo matemáticocomputacional de sistemas en crecimiento que permite de modo sencillo y pedagógico introducir conceptos económicos sobre la base de fenómenos ecológicos. Se realiza una revisión de algunos modelos determinísticos y se confecciona la codificación en Maple ${ }^{\circledR}$, del modelo LotkaVolterra, para el caso particular depredador-presa. Los resultados son relativamente fáciles de obtener, comparado con otros modelos más complejos disponibles en la literatura, el modelo resulta suficientemente estable, y su implementación muestra ser apropiada para los fines propuestos.

Palabras clave: software educativo, modelos ecológicos, modelos económicos, Lotka-Volterra

\section{Computational Analysis of Biological Models for their Application to Economic Models}

\begin{abstract}
The link between the dynamics of the economy and that of ecological populations is analyzed and an educational application is developed. A mathematical-computational model for systems during their growing process, which allows introducing economic concepts on the basis of ecological phenomena, is proposed. A review of some deterministic models is done and the Lotka-Volterra model for the predator-prey case is implemented in Maple ${ }^{\circledR}$. The results are relatively easy to obtain, compared with other more complex models available in the literature, the model is sufficiently stable and its implementation shows to be appropriate for the purposes of this work.
\end{abstract}

Keywords: educational software, ecological models, economic models, Lotka-Volterra 


\section{INTRODUCCION}

Se han propuesto distintas teorías para la explicación de los fenómenos de evolución económica. Sin embargo y hasta ahora, los enfoques simplificados dominan en dichas teorías. Como regla, el mayor acento se da sobre las condiciones de existencia y estabilidad de estados estables. Desafortunadamente, en la economía real estos tipos de comportamiento prácticamente no existen. En general se observa una dinámica compleja: ciclos periódicos, fluctuaciones y caos, ("catástrofe", "bifurcación", "ciclo límite", "atractor extraño", "estructura disipativa", etc). Sobre esta base, hace alrededor de veinte años, se formularon nuevos enfoques dinámicos (Zhang, 1991).

Si bien lo apropiado es estudiar enfoques dinámicos, el desarrollo de teorías económicas demostró que el análisis de los estados de equilibrio puede ser útil a pesar de sus restricciones. Los modelos estáticos, aunque obsoletos, resultan importantes para comprender el funcionamiento de los dinámicos, pero son suficientemente conocidos como para obviar su análisis en esta presentación. El foco principal de la teoría económica moderna es la explicación de los desplazamientos en la producción, de los lugares de trabajo y de los capitales en una economía en crecimiento; así como la determinación de la distribución de ingresos entre los participantes del proceso productivo, cuestiones en las que suelen ser útiles los modelos ecológicos, que actualmente se utilizan en sociología, particularmente en economía.

Hay características comunes que son propias de distintos procesos. Por ejemplo, la oscilación de poblaciones de tipo biológico y de ciclos económicos se describe con sistemas de ecuaciones diferenciales similares. Por ello se considera importante el estudio de modelos ecológicos y su forma de implementación, con el fin de facilitar la comprensión de modelos económicos y aplicar este criterio como herramienta pedagógica en los procesos educativos. Este pensamiento constituye la base para el desarrollo del presente trabajo, cuyos resultados no están dirigidos a economistas, sino a docentes y alumnos que deben comprender y emplear conceptos de fenómenos económicos, sin ser ésa su especialidad. El modelo Depredador-Presa, del modo implementado, es muy útil para analizar la sensibilidad del sistema y ensayar en forma sencilla y atractiva la elección de parámetros. También resulta útil para estudiar tensiones sociales, migración, PBI, evolución de la población, etc.

Para lograr sencillez y efectividad en la implementación del modelo, se utiliza un software de cálculo simbólico (en este caso Maple ${ }^{\circledR}$ ), ya que evidentemente sería inapropiado utilizar un lenguaje de programación de alto nivel de propósito general (Fórum Tecnológico, 2007). Algunos trabajos publicados, de estudios similares, muestran que puede resultar innecesariamente extensa y complicada la implementación recurriendo a lenguajes convencionales de alto nivel (Meza, 1997).

El trabajo resultó de gran utilidad para avanzar hacia un Modelo de la Dinámica Urbana de San Juan-Argentina, ajustando los parámetros mediante el uso de datos estadísticos de esta provincia (Gómez y Nagornov, 2002). Sus aplicaciones son diversas, por ejemplo también puede utilizarse para examinar, como modelo de competición, la sustitución de tecnologías maduras por nuevas (Morris y Pratt, 2003). La herramienta es de rápida implementación; fácil de usar y didáctica para los alumnos en ingeniería de gestión, que no requiere cálculos ni metodologías complejas.

\section{ANTECEDENTES}

A continuación se realiza la revisión de modelos dinámicos de sistemas en crecimiento, clásicos y modernos, de economía y ecología, y se analiza la vinculación entre los económicos y ecológicos.

Modelo de Verhulst-Pearl (ecuación logística)

$$
\frac{d P}{d t}=K P\left(1-\frac{P}{M}\right) \quad x_{n+1}=x_{n}+\beta \cdot x_{n} \cdot \frac{M-X_{n}}{M}
$$

Éste surgió como una crítica al modelo de Malthus y constituyó un claro avance en la modelación. 
Si M es el tamaño límite de la población, es evidente que cuando $P$ es pequeño en comparación con $\mathrm{M}$, la ecuación se reduce a la de Malthus. Por otra parte, si $\mathrm{P}$ toma un valor que tiende a $\mathrm{M}$, el aumento $\mathrm{dP} / \mathrm{dt}$ tiende a cero. Cuanto más se acerca la variable a su tamaño límite, más dificultoso se torna su crecimiento.

\section{Modelo de Haavelmo}

Supuso el siguiente modelo macroeconómico, mediante las ecuaciones (2):

$$
\left.\begin{array}{l}
\frac{d N}{d t}=N\left(a-\frac{\beta N}{Y}\right), a, \beta>0, \\
Y=A N^{\alpha}, \quad A>0,0<\alpha<1
\end{array}\right\}
$$

donde $\mathrm{N}$ es la población, $\mathrm{Y}$ es la producción, $\alpha, \beta, a, A$ son constantes. El reemplazo de la segunda ecuación en la primera resulta en la ecuación número (3):

$$
\frac{d N}{d t}=N\left(a-\frac{\beta N^{1-\alpha}}{A}\right)
$$

Por lo tanto, la ley de variación de $\mathrm{N}$ es la conocida curva logística usada en la teoría de las poblaciones biológicas y de análisis económico. La dinámica de este sistema es muy simple: dependiendo del valor inicial, $N$ va monótonamente a su valor de equilibrio (Véase Fig. 1).

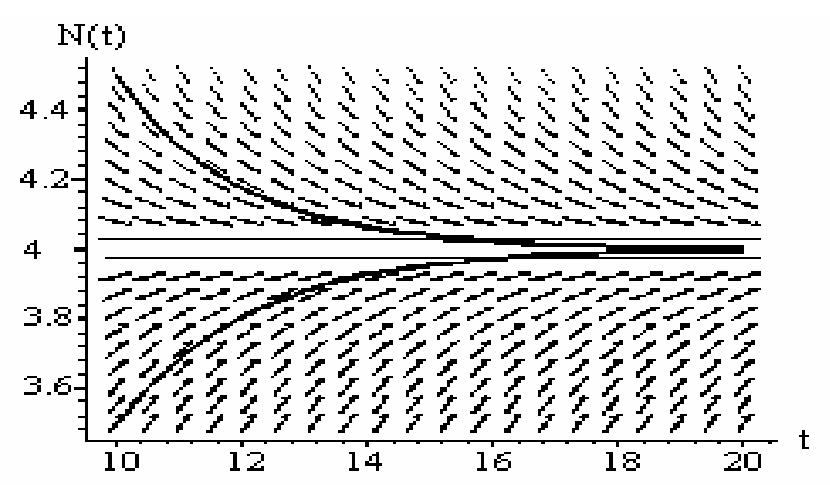

Fig. 1: Puntos de equilibrio del Modelo de Haavelmo

Modelo de Verhulst (modelo de crecimiento compensatorio)

$$
C_{n+1}=2 \cdot C_{n} \quad C_{n+1}=\frac{2}{1+\frac{C_{n}}{K}} \cdot C_{n}
$$

Este modelo surgió a partir del análisis de una clase de bacteria, llamada histidina auxotrophs, que no es capaz de producir su propia histidina (aminoácido necesario para construir las proteínas que son esenciales para la reproducción celular). Cuando estas bacterias se cultivaron en un medio que contenía suficiente histidina, se duplicó su cantidad por división cada 40 minutos. Este caso es de tener en cuenta porque aún con suficiente histidina, aparecen otros factores inter e intraespecíficos que dificultan tal duplicación. Esta consideración es particularmente aplicable y a tener en cuenta en la economía, puesto que variables inter o intraespecíficas, tales como el aumento de oferta de mano de obra, disminución del mercado laboral o importación de productos a bajo precio, influyen fuertemente en la variación de la población de una ciudad, dificultando su crecimiento. 


\section{Caos en el modelo de comercio internacional}

Lorenz propuso el siguiente modelo, que muestra los factores de surgimiento del atractor extraño, que aparece debido al comercio internacional entre economías con ciclos límites:

$$
\left\{\begin{array}{l}
\frac{d x}{d t}=\sigma(y-x), \\
\frac{d y}{d t}=r x-y-x z, \\
\frac{d z}{d t}=x y-b z .
\end{array}\right.
$$

Resolviendo el sistema (5), codificado en Maple ${ }^{\circledR}$, se observa el atractor extraño de Lorenz (solución caótica), que se muestra en la Fig. 2, en tanto que en la Fig. 3 se puede ver en función del tiempo.

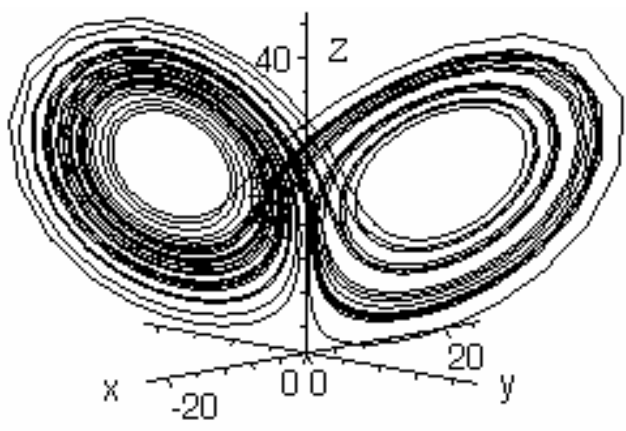

Fig. 2: Atractor Extraño de Lorenz.

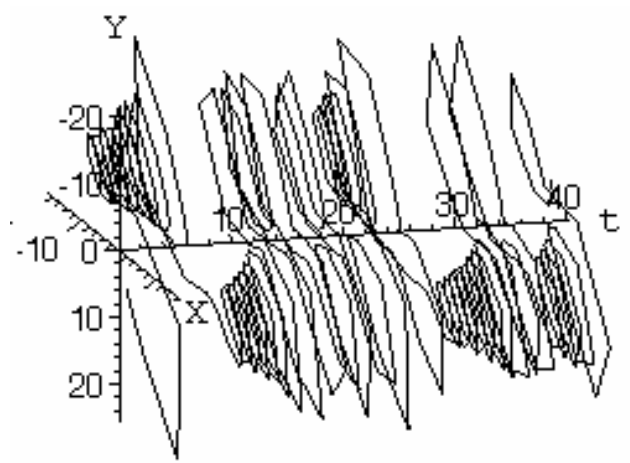

Fig. 3: Atractor Extraño de Lorenz en función del tiempo

\section{Dinámica urbana}

Muestra que se puede observar un comportamiento no regular (atractor extraño) en sistemas dinámicos tridimensionales muy simples.

Considérese la ciudad rodeada por el área metropolitana de forma que resulte pequeña en comparación con ésta. Las características locales de la ciudad se expresan con las tres variables principales siguientes:
$\mathrm{X} \quad$ producción de la ciudad;
Y población de la ciudad;
Z renta de la tierra (inmobiliaria)

La dinámica de la ciudad se describe mediante el sistema de ecuaciones (6), que codificado para Maple ${ }^{\circledR}$ permite estudiar la posible evolución del tamaño de la población y del producto bruto interno (PBI), y la elección de estrategias de control, modificando los parámetros $a_{i}, c_{i}, d_{i}$ :

$$
\left.\begin{array}{l}
\frac{d X}{d t}=a_{1}\left(a_{2} Y-a_{3} X\right), \\
\frac{d Y}{d t}=c_{1}\left(c_{2} X-c_{3} Y\right)-c_{4} X Z, \\
\frac{d Z}{d t}=d_{1} X Y-d_{2} Z,
\end{array}\right\}
$$


Después de la transformación a variables canónicas, resulta el sistema de ecuaciones (5) de Lorenz (Gómez y Nagornov, 2002).

Modelo de Hutchinson o Lotka-Volterra (o modelo de interacción interespecífica)

Una comunidad se compone de todas las especies de plantas y animales que habitan una misma zona. Teniendo en cuenta el número de especies como una mera magnitud, es imposible analizar las complejas interacciones entre todas las especies en una comunidad en particular. El ecologista, por lo tanto, se ha concentrado en el análisis de las interacciones entre dos (a veces tres) especies. Estas interacciones se resumen en la Tabla 1, según sea positivo o negativo el efecto de una especie cualquiera, sobre la otra. La ausencia de efecto se denota con 0 (Morris y Pratt, 2003).

Tabla 1: Interacción entre dos especies

\begin{tabular}{|l|c|c|}
\hline Tipo de Interacción & Efecto Sobre la Especie 1 & $\begin{array}{c}\text { Efecto Sobre la } \\
\text { Especie 2 }\end{array}$ \\
\hline Neutralismo & 0 & 0 \\
\hline Comensalismo & + & 0 \\
\hline Amensalismo & - & 0 \\
\hline $\begin{array}{l}\text { Protocooperación (no } \\
\text { obligatoria) }\end{array}$ & + & + \\
\hline Mutualismo (obligatorio) & + & + \\
\hline Predación & + (depredador) & - (presa) \\
\hline Parasitismo & + (parásito) & - (huésped) \\
\hline Competición & - & - \\
\hline
\end{tabular}

El signo positivo o negativo indica el efecto que una especie tiene sobre la tasa de crecimiento de otra. Sólo dos tipos de interacción se han estudiado en profundidad, desde el punto de vista de la dinámica de la población.

El efecto inhibitorio que cada una de dos especies competitivas tiene en el crecimiento de la población de la otra se ha modelado como una extensión de la ecuación logística, requiriendo así dos ecuaciones de crecimiento, una para cada especie: sistema de ecuaciones (7).

$$
\left.\begin{array}{l}
\frac{d N_{1}}{d t}=r_{1} N_{1}\left(K_{1}-N_{1}-a \frac{N_{2}}{K_{1}}\right) \\
\frac{d N_{2}}{d t}=r_{2} N_{2}\left(K_{2}-N_{2}-b \frac{N_{1}}{K_{2}}\right)
\end{array}\right\}
$$

Los términos de este modelo, Ilamado de Lotka-Volterra, son los mismos del modelo logístico, con el agregado de $a, b$ y los coeficientes de competición que tienen en cuenta los efectos de un individuo de una especie sobre los de la otra. Los supuestos subyacentes a este modelo de competencia son los mismos que para el modelo logístico, con la diferencia que los coeficientes de competición no varían con la densidad de la población. Tratándolo como el caso de dos especies que ocupan el mismo nicho, se pueden analizar tensiones sociales; lo cual se pone de manifiesto en el sistema (9) donde se trata la lucha de clases sociales.

\section{HERRAMIENTAS Y METODOS}

\section{La revolución en ecología y dinámica de población. Vinculación con modelos económicos}

Al principio se hizo referencia a la vinculación entre modelos económicos y biológicos. Las últimas décadas han visto cambios drásticos en ecología teórica. Además de la presentación de nuevas técnicas matemáticas, ha cambiado aún hasta el enfoque filosófico para el entendimiento de la 
dinámica de ecosistemas y poblaciones ecológicas. Nuevos enfoques matemáticos han transformado la disciplina. Los diagramas de flujo y ecuaciones diferenciales dejadas por los pioneros en modelación ecológica han llevado a bifurcaciones, atractor extraños y a trayectorias confusas, que resultan de suma utilidad para los modelos económicos.

Estabilidad estructural de los modelos económicos

Algunas veces es posible encontrar cómo los sistemas dinámicos reaccionan a la perturbación en las condiciones iniciales. Pero en los otros casos interesa estudiar las propiedades funcionales del sistema dinámico mismo. Las propiedades cualitativas de las funciones pueden ser entendidas desde el punto de vista del concepto de estructura de la estabilidad. Los modelos económicos se construyen para explicar los fenómenos auténticos de la vida real. Sin embargo, el resultado puede ser muy sensible a pequeños cambios en el modelo. En este caso, cualquier pequeño cambio en el modelo puede resultar en otro modelo con propiedades significativamente distintas. Para ilustrar el concepto de estabilidad estructural, se revisa el bien conocido modelo biológico Depredador-Presa. Este modelo también fue considerado como económico por muchos economistas y, como veremos, el de Goodwin, permite encontrar puntos comunes con él y vincular los fenómenos de la lucha entre clases y las sociedades biológicas.

Modelo Depredador- Presa

El sistema Depredador-Presa consiste en un sistema de ecuaciones diferenciales ordinarias:

$$
\left\{\begin{array}{l}
\frac{d x}{d t}=\alpha\left(y_{1}-y\right) x \\
\frac{d y}{d t}=\beta\left(x-x_{1}\right) y
\end{array}\right\}
$$

Zhang (1988), Dendrinos (1990) y Mullally (1985) utilizaron este modelo para describir la dinámica de las pequeñas ciudades. La variable $\mathrm{x}$ indica la densidad de uso de terreno, y es la renta del suelo (tierra); $\alpha, \beta, x_{1}, y_{1}$ son parámetros que se estudian oportunamente. El sistema describe el modelo de la oferta-demanda de la renta inmobiliaria especulativa, teniendo en cuenta los futuros porcentajes bajo las expectativas de los propietarios y de los usuarios de las tierras.

La otra aplicación del modelo de Depredador-Presa fue realizada por Goodwin (1951), Gabish y Lorenz (1986). Este modelo fue adoptado para describir la lucha de clases sociales. Se supone la existencia de dos tipos de ciudadanos: los trabajadores y los capitalistas. Los trabajadores gastan todos sus ingresos $w L$ en consumo. Los capitalistas acumulan sus ingresos $Y-w L$, donde $Y$ es la producción. Con transformaciones adecuadas, el modelo de Goodwin puede escribirse de la siguiente manera:

$$
\left.\begin{array}{l}
\frac{d x}{d t}=x\left[\frac{1}{k}-g-n-\frac{y}{k}\right] \\
\frac{d y}{d t}=y[-g-r+b x]
\end{array}\right\}
$$

Analizando estas ecuaciones se observa que el modelo de Goodwin coincide con el modelo Depredador-Presa. Por lo tanto, las propiedades de éste último serán consideradas en detalle posteriormente. La analogía de dicho modelo y el de Goodwin nos permite encontrar los puntos comunes entre los fenómenos de la lucha entre clases y las sociedades biológicas. Este modelo es relativamente simple y puede resultar en efectos oscilatorios. Sin embargo, su aplicación se ve restringida debido a su inestabilidad a pequeños cambios de forma funcional que influencian en las propiedades cualitativas del sistema. Es muy importante encontrar tales propiedades del modelo que no sean sensibles a pequeños cambios en el mismo.

\section{Caso particular del modelo de Hutchinson o Lotka-Volterra}

Aplicación de la interacción de dos especies: Zorros Vs. Conejos. 
Se definen las siguientes constantes y variables:

$r$ : número de conejos, $f$ : número de zorros, $\alpha_{1}$ : coeficiente de interacción, $\gamma_{1}$ : coeficiente de crecimiento de los conejos, $\alpha_{2}$ : coeficiente de interacción, $\gamma_{2}$ : coeficiente de decaimiento de los zorros

$$
\left.\begin{array}{l}
\frac{\partial r(t)}{\partial t}=\gamma_{1} r(t)-\alpha_{1} r(t) f(t) \\
\frac{\partial f(t)}{\partial t}=-\gamma_{2} f(t)+\alpha_{2} r(t) f(t)
\end{array}\right\}
$$

La siguiente codificación para Maple ${ }^{\circledR}$ resuelve el sistema (10) para el conjunto de parámetros cuyo resultado se muestra en la Fig. 4:

restart:

unprotect(gamma); \# Otherwise gamma is Euler's constant.

sys:=

$\operatorname{diff}(r(t), t)=$ gamma ${ }^{*} r(t)-$ alpha1 ${ }^{*} r(t)^{*} f(t)$,

$\operatorname{diff}(\mathrm{f}(\mathrm{t}), \mathrm{t})=-$ gamma2* $\mathrm{f}(\mathrm{t})+$ alpha2* $\mathrm{r}(\mathrm{t})^{*} \mathrm{f}(\mathrm{t})$;

sys;

gamma1:=2;gamma2:=1;alpha1:=.01;alpha2:=0.01;

$$
\begin{aligned}
& \text { vars: }=\{r(t), f(t)\} ; \\
& \text { ic: }=r(0)=100, f(0)=5 ;
\end{aligned}
$$

sol:=dsolve(\{sys,ic\}, vars, numeric, output=listprocedure);

with (plots):

odeplot(sol, $[\mathrm{t}, \mathrm{r}(\mathrm{t}), \mathrm{f}(\mathrm{t})], 0 . .10$, axes=normal, numpoints $=200$, labels $=[\mathrm{t}, \mathrm{r}, \mathrm{f}]$, color=blue,

title='A graph of foxes (f) vs rabbits $(r)^{\prime}$, orientation=[0,90],tickmarks=[3,3,3]);

\section{RESULTADOS Y DISCUSIÓN}

En el programa elaborado en Maple ${ }^{\circledR}$ para resolver el sistema de ecuaciones diferenciales (10), se asignan valores a los parámetros valores escogidos empíricamente (Morris y Pratt, 2003), a partir de numerosos ensayos, para poner de manifiesto adecuadamente las propiedades y funcionamiento del modelo: $\gamma_{1}=2 ; \gamma_{2}=1 ; \alpha_{1}=.01 ; \alpha_{2}=.01$. Ejecutado el programa, se observa que con muy pocos zorros en el nicho ecológico, no más de 8 o 10, los conejos aumentan la población rápidamente hasta alcanzar aproximadamente los 840 hacia la mitad de la segunda unidad de tiempo. Mientras que los zorros han ido creciendo lentamente durante la primer unidad de tiempo y ya, con suficientes recursos, aumentan bruscamente hasta alcanzar los 1.080 ejemplares sobre el final de la segunda, donde se encuentran en su apogeo (Ver diferentes vistas en la Fig. 4).
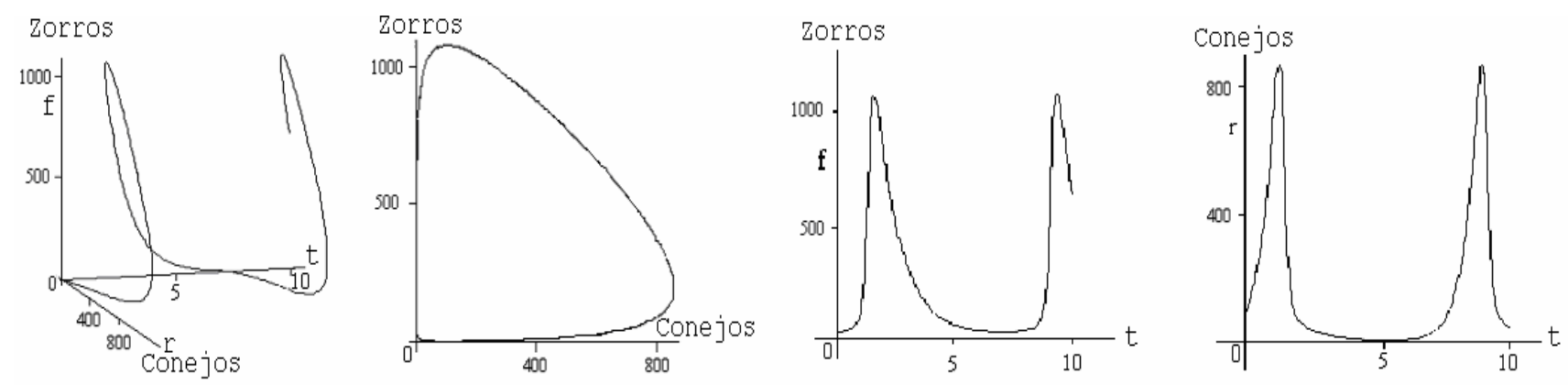

Fig. 4: Distintas vistas de la Dinámica zorros, conejos, tiempo.

Esto ha ocurrido a expensas de que los conejos han disminuido hasta 20 o 30 al final de la segunda unidad. Los zorros ya con muy pocos conejos, vuelven a decaer. Pero, con un bajo $\mathrm{Y}_{2}$, disminuyen lentamente y tardan alrededor de 7 unidades de tiempo para ponerse cerca de la extinción. En este punto, puede suponerse que los zorros no se alimentan solo de conejos ya que de otro modo no podrían sobrevivir entre el final de la segunda unidad de tiempo y la séptima, lapso en el que la población de conejos es muy baja y casi no se producen encuentros entre 
ambas especies. También puede suponerse que han inmigrado algunos conejos de otras regiones. Casi extinguidos las zorros, entre la séptima y octava unidad de tiempo los conejos prosperan libremente y se inicia un nuevo ciclo, en el que empieza a aumentar el número de zorros, puesto que los encuentros ocasionales comienzan a ser más frecuentes. Para los valores asignados a los parámetros, el sistema es estable.

Dando los valores $\gamma_{1}=2 ; \gamma_{2}=1 ; \alpha_{1}=1 ; \alpha_{2}=1$ el modelo responde como se muestra en la Fig. 5 .

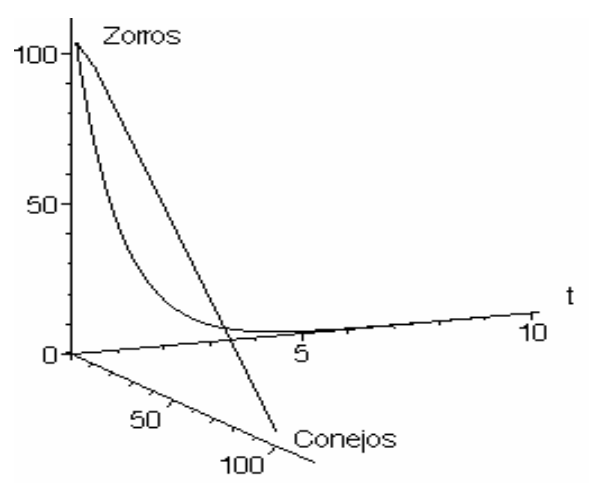

Fig. 5: Dinámica zorros, conejos, tiempo.

Con estos valores asignados a las constantes, se observa que los conejos crecen hasta 100 y son consumidos muy rápidamente por los zorros, que alcanzan también aproximadamente 100 . Como $\alpha_{1}$ y $\alpha_{2}$ han sido aumentados 100 veces, la interacción entre ambas especies se torna muy elevada, es decir, comienza a tener un fuerte peso el segundo sumando en ambas ecuaciones, que equivale a una fuerte interacción entre ambas especies. Al quedarse sin conejos, los zorros disminuyen su número en forma relativamente lenta.

Como consecuencia desaparecen ambas especies ya que no logran recuperarse y el sistema es inestable. Esto se ha comprobado con 30 períodos de tiempo y el sistema sigue resultando inestable.

Observación: Para profundizar el caso se realiza la prueba de dejar constante $\gamma_{1}=2$ y se aumenta 5 veces $\gamma_{2}$, es decir $\gamma_{2}=5$. Si además, para tener una mejor visión, se aumenta a 20 el número de unidades de tiempo, como se muestra en la Fig. 6, se concluye: Los zorros decrecen mucho más rápidamente con lo que se salva algún par de parejas de conejos y algún par de parejas de zorros. Como las características del nicho ecológico lo permiten, estos pocos sobrevivientes de cada especie no se encuentran con los de la otra, lo que impide la interacción. En estas circunstancias, los conejos prosperan muy lentamente hasta el comienzo de la novena unidad de tiempo, que es cuando su población crece a 100 bruscamente en un corto lapso, lo cual, a su vez, es conducente a que se produzcan encuentros con los pocos zorros que quedan. Ello, desde luego, es motivo para que la población de los zorros crezca con elevada rapidez, dado que al aumentar la población de conejos adquiere importancia el segundo sumando de la segunda ecuación. Los zorros aumentan a expensas de los conejos que disminuyen en ese caso. Se inicia un nuevo ciclo y el sistema se torna estable.

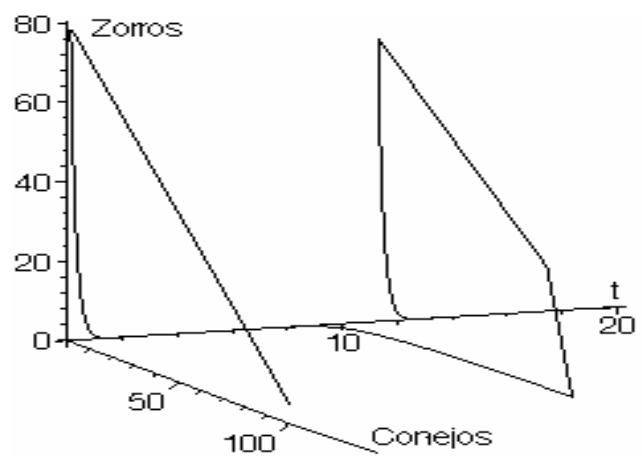

Fig. 6: Dinámica zorros, conejos, tiempo. 
Sintetizando, respecto a los conejos: Un elevado valor de $\gamma_{1}$ provoca un rápido crecimiento de los conejos. Esto, obviamente, se ve influenciado por el número de conejos y de zorros en un momento dado, cuyo producto es afectado por el coeficiente $\alpha_{1}$ que, como era de esperar del análisis de las ecuaciones, mientras más alto sea, más interferirá en el crecimiento de los conejos. Además es claro, que para un mismo $\alpha_{1}$, mientras mayor sea la población de zorros, el crecimiento de los conejos se tornará más lento. Lo mismo puede decirse que, a igual número de zorros, a mayor $\alpha_{1}$, más se entorpece el crecimiento de los conejos.

Con relación a los zorros: Con un elevado $\gamma_{2}$ el decaimiento de los zorros es muy rápido, tanto más rápido cuanto mayor es $\gamma_{2}$. Para un mismo $\alpha_{2}$, el crecimiento de los zorros será más rápido cuanto mayor sea la población de conejos. Para una misma cantidad de conejos, el crecimiento de los zorros será tanto más elevado cuanto más grande sea $\alpha_{2}$. Ello permite colegir que se pueden escoger estrategias de control de modelos biológicos tales como bacterianos, cáncer, especies de plantas (Lenhart y Workman, 2007), actuando sobre los valores de estos parámetros, cuando el modelo se utiliza para estudiar tensiones sociales, migración, PBI, evolución de la población, donde, obviamente, se trata de modificar parámetros económicos.

Con relación a aplicaciones económicas y/o de gestión se ha comprobado, en estudios separados, que el sistema (6), conformado por el mismo tipo de ecuaciones, presenta un buen comportamiento para analizar la dinámica de una ciudad (específicamente para la provincia de San Juan - Argentina) en lo referente a la predicción de la evolución del tamaño de la población y del producto bruto interno para los próximos 10 años (Gómez y Nagornov, 2002). Calculando las constantes mediante la representación del sistema EDO en diferencias finitas, con un paso de tiempo de un año, y utilizando las estadísticas de los últimos 10 años de la provincia acerca del producto bruto interno, la población y la renta de la tierra. Gráficamente se observan los resultados en las Fig. 7 y 8.

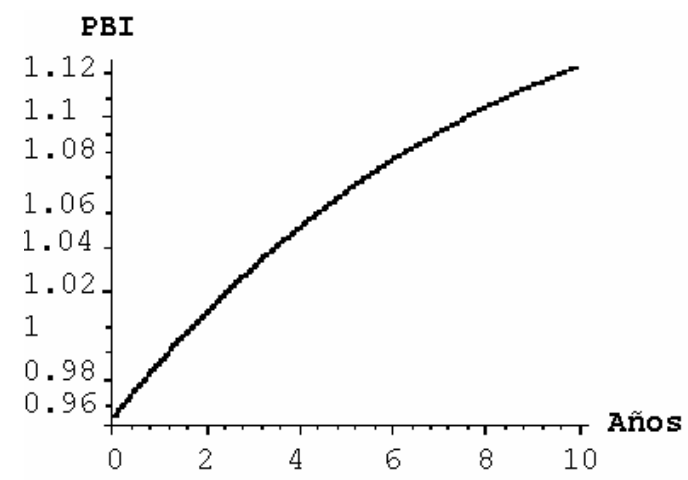

Fig. 7: Crecimiento relativo del PBI

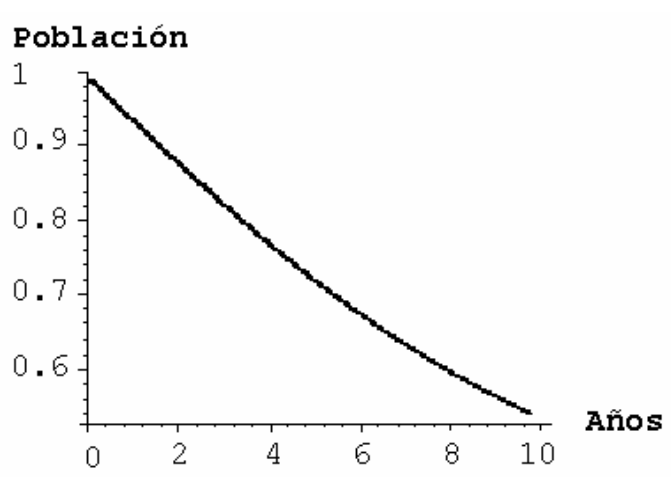

Fig. 8: Probable evolución de la población

\section{CONCLUSIONES}

En los resultados obtenidos, se observa que: 1) Es posible una implementación sencilla del modelo, de operación muy amigable; que permite extraer conclusiones con facilidad: a) Ambas especies influencian en la evolución de si misma y de la otra. b) Existe interacción ínterespecífica e intraespecífica. c) El resultado final del modelo de competición es la eliminación de una especie por la otra. Ambas especies inhiben el crecimiento de la otra por un tiempo, pero finalmente una especie gana, creciendo a expensas de la otra (el funcionamiento del sistema depende de las condiciones del nicho ecológico). La especie ganadora es finalmente limitada por su propio índice de tamaño o límite de la población (ver modelo Logístico o de Verhulst-Pearl), mientras que la especie perdedora se extingue. Se logra observar claramente el denominado Principio de Exclusión o axioma de Gauss, que puede resumirse de la siguiente manera: Dos especies no pueden ocupar indefinidamente el mismo nicho ecológico, pues el resultado inevitable es que una especie eliminará a la otra puesto que ambas usan la misma cantidad limitada de recursos ambientales de igual manera. 2) El modelo es suficientemente estable y sensible a las variaciones de los parámetros encontrados (pudiéndose controlar fácilmente variándolos), y se comporta con adecuado equilibrio si se eligen apropiadamente sus valores. 3) El modelo computacional permite observar de modo sencillo y ameno la dinámica de poblaciones 
ecológicas. También constituye una interesante herramienta didáctica para ser utilizada con fines educativos, estudiar tensiones sociales, migración, etc., y en gestión, para la elección de estrategias de control.

\section{REFERENCIAS}

Fórum Tecnológico, Addlink Software Científico S.L., CIF B-59852053, Maple 11 y el proceso de diseño en ingeniería: Matemáticas, Modelado y Simulación [en línea]. Barcelona, España, $\begin{array}{llllll}\text { Número } & 10 & \text { (2007), } & \text { [Citado Julio 20, }\end{array}$ http://www.forumtecnologico.net/articulos html/ft $10 \mathrm{html} / 10-11 /$ index.asp

Gómez, R. y O. Nagornov; Study of Urban Dynamics of San Juan by the Lorenz Model. Proceedings Of International Scientific Session Mephi, Moscú, Federación Rusa. V 7. Edición 60, pp 77-78 (2002). http://www.mephi.ru

Lenhart, Suzanne y John T. Workman; Optimal control applied to biological models, Chapman \& Hall/Crc Mathematical and Computational Biology, Portland, USA (2007).

Meza, Adolfo Castillo; Modelos Matemáticas para la Ecología, Economía y Derecho Ambiental, Resolución Numérica de Ecuaciones de Lotka-Volterra, Departamento de Física y Matemáticas Universidad Peruana Cayetano Heredia, [Citado 5 agosto 2009], 1997. Url: http://www.upch.edu.pe/facien/virtual/lotka.htm

Morris, Steven A. y David Pratt; Analysis of the lotka-volterra competition equations as a technological substitution model. Technological Forecasting and Social Change: 70(2), 103-133 (2003).

Zhang, W.; Synergetic economics. Time and Change in nonlinear economic. SpringuerVerlag. Berlin-Heidelberg (1991). 